\title{
Generalized linkage construction for constant-dimension codes
}

\author{
Daniel Heinlein*
}

November 14, 2019

\begin{abstract}
A constant-dimension code (CDC) is a set of subspaces of constant dimension in a common vector space with upper bounded pairwise intersection. We improve and generalize two constructions for CDCs, the improved linkage construction and the parallel linkage construction, to the generalized linkage construction which in turn yields many improved lower bounds for the cardinalities of CDCs; a quantity not known in general.
\end{abstract}

Keywords: Finite projective spaces, constant-dimension codes, subspace codes, subspace distance, rank distance, maximum rank-distance codes, lifted maximum rank-distance bound, combinatorics.

MSC classification: 51E20; 94B65, 05B25.

\section{Introduction}

Let $V \cong \mathbb{F}_{q}^{v}$ be a $v$-dimensional vector space over a finite field with $q$ elements $\mathbb{F}_{q}$. The set of all subspaces of $V$ forms a metric space with respect to the so-called subspacedistance $\mathrm{d}_{\mathrm{s}}(U, W)=\operatorname{dim}(U+W)-\operatorname{dim}(U \cap W)$ cf. [21, Lemma 1]. A $(v, N, d ; k)_{q}$ constant-dimension code (CDC) is a set of $k$-subspaces of $V$ of cardinality $N$ such that the subspace-distance of each pair of distinct elements, called codewords, is at least $d$.

Coding in this metric space was motivated by Kötter and Kschischang in 21. The main question of subspace coding in the constant-dimension case asks for the maximum cardinality $N$ of a $(v, N, d ; k)_{q}$ code. This maximum cardinality is denoted as $A_{q}(v, d ; k)$. The homepage http://subspacecodes.uni-bayreuth.de/, see also the manual [19], lists the currently best known lower and upper bounds on $A_{q}(v, d ; k)$ for $q \leq 9, v \leq 19$, all $d$, and all $k$.

Many good lower bounds for CDCs arise from linkage type constructions; Section 4 provides an overview. This paper generalizes two already successful constructions, the

${ }^{*}$ D. Heinlein is with the Department of Communications and Networking, Aalto University, Finland, e-mail: firstname.lastname@aalto.fi. 
improved linkage construction (Theorem 17) and the parallel linkage construction (Theorem 201), to the so-called generalized linkage construction (Theorem 25).

According to the ranking in the homepage, the improved linkage construction is among the best known constructions in $\approx 50.7 \%$ of the listed parameters while the parallel linkage construction is among the best known constructions in $\approx 6.3 \%$ of the listed parameters. The generalized linkage construction is among the best known constructions in $\approx 52.5 \%$ of the listed parameters.

As these numbers change if new bounds are introduced in the database, especially since most linkage type constructions refer back to smaller CDCs as building blocks, we prove in Lemma 28 and Lemma 29 that the generalized linkage construction is strictly better for an infinite family of parameters than the parallel linkage construction and the improved linkage construction, respectively.

The rest of the paper is organized as follows. In Section 2, we introduce the notation used, in particular $q$-binomial coefficients, rank-metric codes and their sizes, and bounds needed for the comparison of the linkage constructions. We need rank-metric codes having the additional property that each codeword has an upper bounded rank.

Bounds for the cardinalities of these rank-restricted rank-metric codes and special cases are determined in Section 3 .

Section 4 provides an overview over two families of linkage type constructions. Both families are generalized in a single construction in Section 5. For some parameters, the new construction is strictly better, as shown in Section 6.

\section{Preliminaries}

Throughout the paper, we will use the following notation and facts about $q$-binomial coefficients. For prime powers $q \geq 2$ and $0 \leq n$ we use the $q$-numbers $[n]_{q}=\left(q^{n}-\right.$ 1) $/(q-1)=\sum_{i=0}^{n-1} q^{i}$, the $q$-factorials $[n]_{q} !=\prod_{i=1}^{n}[i]_{q}$, and the $q$-binomial coefficients $\left[\begin{array}{l}v \\ k\end{array}\right]_{q}=\frac{[v]_{q} !}{[k]_{q} ![v-k]_{q} !}=\prod_{i=0}^{k-1} \frac{q^{v}-q^{i}}{q^{k}-q^{i}}$ for $0 \leq k \leq v$. An empty sum is defined to be 0 and an empty product is 1 , so that $[0]_{q}=0$ and $[0]_{q} !=1$ in particular. Note that $[n]_{q}<q^{n}$. The set of $k$-subspaces in $\mathbb{F}_{q}^{v}$ is denoted as $\left[\begin{array}{c}\mathbb{F}_{q}^{v} \\ k\end{array}\right]$ and its cardinality is $\left[\begin{array}{l}v \\ k\end{array}\right]_{q}$.

The sizes of $q$-binomial coefficients can be estimated with

Lemma 1 ( [21, Lemma 4], cf. [17, Lemma 8]). For all prime powers $q \geq 2$ and $0<k<v$, we have

$$
1<\left[\begin{array}{l}
v \\
k
\end{array}\right]_{q} / q^{k(v-k)}<\left(\prod_{i=1}^{\infty}\left(1-q^{-i}\right)\right)^{-1}<3.47 .
$$

We use the following well known connection between subspaces and full-rank matrices. The reduced row echelon form of the matrix $A$ is denoted as $\mathrm{R}(A)$. Then, the bijection between subspaces and their canonical basis in reduced row echelon form, written as rows of a matrix, is

$$
\tau:\left[\begin{array}{c}
\mathbb{F}_{q}^{v} \\
k
\end{array}\right] \rightarrow\left\{A \in \mathbb{F}_{q}^{k \times v} \mid \operatorname{rk} A=k \wedge A=\mathrm{R}(A)\right\},
$$


in particular, $U$ is the row-span of $\tau(U)$ for any subspace $U$. We omit the dependency of $\tau$ on $q, k, v$ as the context determines them and we extend the codomain of $\tau(\cdot)$ by $\tau^{-1}(A)=\tau^{-1}(\mathrm{R}(A))$ for any matrix $A$ of full row-rank.

For a matrix $A$ in reduced row echelon form, $\mathrm{p}(A)$ is the binary vector with $\mathrm{p}(A)_{i}=1$ iff column $i$ is a pivot column in the matrix $A$. We extend the domain of $\mathrm{p}(\cdot)$ by $\mathrm{p}(B)=\mathrm{p}(\mathrm{R}(B))$ for any matrix $B$ and $\mathrm{p}(U)=\mathrm{p}(\tau(U))$ for any subspace $U$.

The horizontal concatenation of matrices or vectors $A, B$ of compatible sizes and ambient fields is denoted as $A \mid B$.

In addition to the subspace-distance

$$
\begin{aligned}
& \mathrm{d}_{\mathrm{s}}(U, W) \\
= & \operatorname{dim}(U+W)-\operatorname{dim}(U \cap W) \\
= & \operatorname{dim}(U)+\operatorname{dim}(W)-2 \operatorname{dim}(U \cap W) \\
= & 2 \operatorname{dim}(U+W)-\operatorname{dim}(U)-\operatorname{dim}(W) \\
= & 2 \operatorname{rk}\left(\begin{array}{c}
\tau(U) \\
\tau(W)
\end{array}\right)-\operatorname{dim}(U)-\operatorname{dim}(W)
\end{aligned}
$$

for two subspaces $U, W$ of a common vector space, we will also need the Hammingdistance $\mathrm{d}_{\mathrm{h}}(u, w)=\#\left\{i \mid u_{i} \neq w_{i}\right\}$ of two vectors $u, w$ in a common vector space, in particular the weight of $u$ defined as $\mathrm{w}(u)=\mathrm{d}_{\mathrm{h}}(u, 0)$, and the rank-distance $\mathrm{d}_{\mathrm{r}}(A, B)=$ $\operatorname{rk}(A-B)$ of two matrices $A, B$ of compatible sizes and ambient fields.

It is well known that the Hamming-distance of pivot vectors of subspaces lower bounds their subspace-distance.

Lemma 2 ( [7, Lemma 2]). Let $U$ and $W$ be two subspaces of a common vector space, then

$$
\mathrm{d}_{\mathrm{h}}(\mathrm{p}(U), \mathrm{p}(W)) \leq \mathrm{d}_{\mathrm{s}}(U, W)
$$

We will apply

$$
\mathrm{d}_{\mathrm{h}}\left(u\left|u^{\prime}, v\right| v^{\prime}\right)=\mathrm{d}_{\mathrm{h}}(u, v)+\mathrm{d}_{\mathrm{h}}\left(u^{\prime}, v^{\prime}\right)
$$

for any $q$-ary vectors of compatible lengths and the lower bound

$$
|\mathrm{w}(u)-\mathrm{w}(v)| \leq \mathrm{d}_{\mathrm{h}}(u, v)
$$

for two binary vectors $u, v$ of equal length, as any pivot vector is a binary vector.

We will also make use of

$$
\begin{aligned}
\operatorname{rk}(X) \leq & \operatorname{rk}(X \mid Y) \leq \operatorname{rk}(X)+\operatorname{rk}(Y) \\
& \operatorname{rk}(X+Y) \leq \operatorname{rk}(X)+\operatorname{rk}(Y) \\
\operatorname{rk}(X)+\operatorname{rk}(Y)-n \leq & \operatorname{rk}(X \cdot Y) \leq \min \{\operatorname{rk}(X), \operatorname{rk}(Y)\}
\end{aligned}
$$

for any matrices $X, Y$ of compatible sizes and ambient fields, such that $n$ is the number of columns of $X$. 
A rank-metric code (RMC) is a subset of $\mathbb{F}_{q}^{a \times b}$ of cardinality $N$ such that the rankdistance of each pair of codewords is at least $d$. An RMC is called linear, if it is a subspace of $\mathbb{F}_{q}^{a \times b}$. These parameters are abbreviated as $(a \times b, N, d)_{q}$. If in addition the rank of each codeword is at most $u$, we augment the notation to $(a \times b, N, d ; u)_{q}$ and refer to it as rank-restricted RMC (RRMC). The maximum size of an $(a \times b, N, d ; u)_{q}$ $\mathrm{RMC}$ is denoted as $\Lambda(q, a, b, d, u)$. For a $(k \times n, N, d)_{q} \mathrm{RMC} \mathcal{R}$, the lifted RMC of $\mathcal{R}$ is defined as $\left\{\tau^{-1}(I \mid R): R \in \mathcal{R}\right\}$. It is a $(k+n, N, 2 d ; k)_{q}$ CDC.

Delsarte [6] and Gabidulin [11] determined the maximum cardinality

$$
M(q, a, b, d)=\left\lceil q^{\max \{a, b\}(\min \{a, b\}-d+1)}\right\rceil
$$

of RMCs for all parameters $q, a, b, d$, and $\min \{a, b\} \leq u$ and gave constructions to build bound-achieving RMC codes, so-called maximum rank-distance (MRD) codes.

The theory of Delsarte in [6] allows to determine the rank-distribution in a linear MRD code. In his words, a linear MRD code in $\mathbb{F}_{q}^{a \times b}$ and minimum rank-distance $d$ is equivalent to an $(\min \{a, b\}, \max \{a, b\}, \min \{a, b\}-d+1, q)$-Singleton system and can be seen as a $(d-1)$-codesign of cardinality $q^{\max \{a, b\}(\min \{a, b\}-d+1)}$, which is a set of bilinear forms $X \subseteq\left\{f: \mathbb{F}_{q}^{\min \{a, b\}} \times \mathbb{F}_{q}^{\max \{a, b\}} \rightarrow \mathbb{F}_{q} \mid f\right.$ bilinear form $\}$ such that $\operatorname{rk}(f-g)>d-1$ for all $f \neq g \in X$.

Theorem 3 ( [ㅁ, Theorem 5.6], cf. [5, Corollary 26]). The number of matrices with rank $r(d \leq r \leq \min \{a, b\})$ in a linear MRD code in $\mathbb{F}_{q}^{a \times b}$ and minimum rank-distance $d$ is given by

$$
\begin{aligned}
& D(q, a, b, d, r) \\
= & {[\underset{r}{\min \{a, b\}}]_{q} \cdot \sum_{i=0}^{r-d}(-1)^{i} q^{\left(\begin{array}{l}
i \\
2
\end{array}\right)}\left[\begin{array}{l}
r \\
i
\end{array}\right]_{q}\left(q^{\max \{a, b\}(r-d+1-i)}-1\right) . }
\end{aligned}
$$

We abbreviate

$$
\Delta(q, a, b, d, u)=1+\sum_{i=d}^{\min \{u, a, b\}} D(q, a, b, d, i)
$$

which is the size of the largest subset of a linear MRD code in $\mathbb{F}_{q}^{a \times b}$ and minimum rank-distance $d$ such that the rank of each included matrix is at most $u$.

We deliberately allow matrices with zero rows or zero columns and count sets containing only one such matrix with cardinality one and denote the all-zero matrix with 0 and the identity matrix with $I$.

Theorem 3 and $\Delta(q, a, b, d, u)$ provide only a construction for $(a \times b, N, d ; u)_{q}$ RMC. In fact, we have

$$
\Delta(q, a, b, d, u) \leq \Lambda(q, a, b, d, u) \leq M(q, a, b, d)
$$

The number of matrices of a given rank in a finite vector space is well known. 
Theorem 4 ( [23], [10, Theorem 2]). The number of matrices in $\mathbb{F}_{q}^{a \times b}$ of rank $r$ is

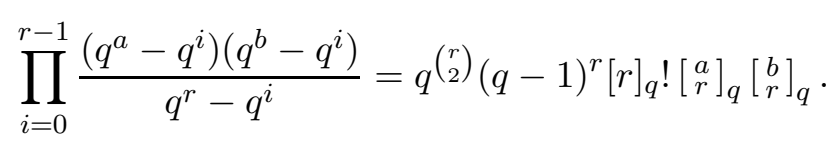

Due to the following lemma, we can without loss of generality restrict the parameters of a CDC to $2 \leq d / 2 \leq k \leq v / 2$, see [17, Page 33f.] or [18, Page 4822] for an extensive discussion.

Lemma 5 ( [21, Page 3582, Equation 4]). For all $q$ and $2 \leq d / 2 \leq k \leq v$, we have

$$
A_{q}(v, d ; k)=A_{q}(v, d ; v-k) .
$$

We will use the following lower bound by Cossidente and Pavese to compare the generalized linkage construction (Theorem 25) to the parallel linkage construction (Theorem 21) in Lemma 28 and to the improved linkage construction (Theorem 17) in Lemma 29.

Theorem 6 ( [4, Theorem 4.3]).

$$
A_{q}(8,4 ; 4) \geq q^{12}+q^{2}\left(q^{2}+1\right)^{2}\left(q^{2}+q+1\right)+1 .
$$

For CDCs having $d=2 k$, i.e., the minimum subspace-distance is as large as possible, and the dimension of the ambient vector space is a multiple of the dimension of the codewords, Beutelspacher showed that there are bound-achieving codes. This setting is often referred to as spread.

Theorem 7 ( [1]). For all $q, 1 \leq k$, and $k \mid v$, we have

$$
A_{q}(v, 2 k ; k)=[v]_{q} /[k]_{q} .
$$

An easy to use yet strong upper bound for CDCs is the so-called Anticode bound.

Theorem 8 ( [26, Theorem 5.2], 9, Theorem 1]). For all $q$ and $2 \leq d / 2 \leq k \leq v$, we have

$$
A_{q}(v, d ; k) \leq\left[\begin{array}{c}
v \\
k-d / 2+1
\end{array}\right]_{q} /\left[\begin{array}{c}
k \\
k-d / 2+1
\end{array}\right]_{q} .
$$

\section{Bounds for $\Lambda$}

Here, we adapt the proof of the upper bound of the size of an MRD code to obtain an upper bound on $\Lambda(q, a, b, d, u)$.

This particular upper bound is the Singleton bound applied to the metric space $\left(\mathbb{F}_{q}^{a \times b}, \mathrm{~d}_{\mathrm{r}}\right)$ via the puncturing operation $g: \mathbb{F}_{q}^{a \times b} \rightarrow \mathbb{F}_{q}^{a \times(b-1)}$ mapping a matrix to its first $b-1$ columns, i.e., it cuts the last column off. 
Theorem 9. Let $2 \leq d \leq \min \{a, b\}$ and $0 \leq u$ be integers. Then, we have $\Lambda(q, a, b, d, u) \leq$ $\Lambda(q, a, b-1, d-1, u)$ and $\Lambda(q, a, b, d, u) \leq$

$$
\sum_{r=0}^{\min \{u, \min \{a, b\}-d+1\}} q^{\left(\begin{array}{c}
r \\
2
\end{array}\right)}(q-1)^{r}[r]_{q} ![\underset{r}{\min \{a, b\}-d+1}]_{q}[\underset{r}{\max \{a, b\}}]_{q} .
$$

Proof. Let $b \leq a$ without loss of generality, otherwise transpose. Note that, for matrices $A$ and $B$ of compatible size and ambient field, $\operatorname{rk}(A)-\operatorname{rk}(g(A)) \in\{0,1\}$ (cf. Inequality (4) ), which implies $\mathrm{d}_{\mathrm{r}}(A, B)-\mathrm{d}_{\mathrm{r}}(g(A), g(B)) \in\{0,1\}$, hence $\mathrm{d}_{\mathrm{r}}(A, B)-1 \leq$ $\mathrm{d}_{\mathrm{r}}(g(A), g(B))$ and $\operatorname{rk}(g(A)) \leq \operatorname{rk}(A)$. So the puncturing operation applied to all elements of an $(a \times b, N, d ; u)_{q}$ RMC yields an $\left(a \times(b-1), N^{\prime}, d-1 ; u\right)_{q} \mathrm{RMC}$ and this is an injective map if $2 \leq d$, i.e., $N^{\prime}=N$. Applying the puncturing operation $d-1$ times yields an $(a \times(b-d+1), N, 1 ; u)_{q}$ RMC $\mathcal{R}$. We have

$$
\mathcal{R} \subseteq\left\{A \in \mathbb{F}_{q}^{a \times(b-d+1)} \mid \operatorname{rk} A \leq u\right\}
$$

and consequently

$$
\Lambda(q, a, b, d, u) \leq \#\left\{A \in \mathbb{F}_{q}^{a \times(b-d+1)} \mid \operatorname{rk} A \leq u\right\} .
$$

Then, Theorem 4 allows to determine the cardinality of the right hand side and to complete the proof.

In addition to the recursion provided in Theorem 9, i.e., $\Lambda(q, a, b+1, d+1, u) \leq$ $\Lambda(q, a, b, d, u)$, a similar argument shows $\Lambda(q, a+1, b, d+1, u) \leq \Lambda(q, a, b, d, u)$ and trivially, we also have $\Lambda(q, a, b, d, u-1) \leq \Lambda(q, a, b, d, u)$.

The bound in Theorem 9 is equivalent to $\Lambda(q, a, b, d, u) \leq M(q, a, b, d)$, cf. Inequality (7), iff $\min \{a, b\}<d+u$ and stronger iff $d+u \leq \min \{a, b\}$.

$\Lambda(q, a, b, d, u)$ is precisely the clique number of a graph with vertex set $\left\{A \in \mathbb{F}_{q}^{a \times b} \mid \operatorname{rk} A \leq u\right\}$ and two vertices $A$ and $B$ share an edge iff $\mathrm{d}_{\mathrm{r}}(A, B) \geq d$. The number of vertices can be computed by Theorem 4 .

Using GAP [13] and Cliquer [24] we compute

$$
\begin{array}{ll}
\Lambda(2,2,2,2,1)=3, & \Lambda(2,3,2,2,1)=3, \\
\Lambda(2,3,3,2,1)=7, & \Lambda(2,3,3,2,2)=50, \\
\Lambda(2,4,4,2,1)=15, & \Lambda(2,4,4,4,2)=5, \\
\Lambda(3,2,2,2,1)=4, & \Lambda(3,3,3,2,1)=13, \text { and } \\
\Lambda(3,4,2,2,1)=4 . &
\end{array}
$$

The bounds of Inequality (7) are

$$
\begin{aligned}
1 & \leq \Lambda(q, 3,3,2,1) \leq q^{6}, \\
q\left(q^{4}+q^{3}+q^{2}-q-1\right) & \leq \Lambda(q, 3,3,2,2) \leq q^{6}, \text { and } \\
q\left(q^{7}+q^{6}+2 q^{5}+q^{4}-q^{2}-2 q-1\right) & \leq \Lambda(q, 4,4,2,2) \leq q^{12} .
\end{aligned}
$$


Theorem 9 implies

$$
\begin{aligned}
& \Lambda(q, 3,3,2,1) \leq q\left(q^{3}+q^{2}-1\right), \\
& \Lambda(q, 3,3,2,2) \leq q^{6}, \text { and } \\
& \Lambda(q, 4,4,2,2) \leq q^{3}\left(q^{7}+q^{6}+q^{5}-q^{4}-q^{3}-q^{2}+1\right) .
\end{aligned}
$$

We can prove basic structure results for RRMCs.

Lemma 10. Let $\mathcal{C}$ be an $(a \times b, N, d ; u)_{q} R M C$ with $2 \leq N$, then $d-u \leq \operatorname{rk}(A) \leq u$ for each matrix $A$ in $\mathcal{C}$, there is at most one matrix $M$ in $\mathcal{C}$ with $\operatorname{rk}(M)<d / 2$, and $d \leq \mathrm{d}_{\mathrm{r}}(X, Y) \leq 2 u$ for all $X \neq Y \in \mathcal{C}$.

Proof. By Inequality (5), we have $d \leq \operatorname{rk}(A-B) \leq \operatorname{rk}(A)+\operatorname{rk}(-B) \leq \operatorname{rk}(A)+u \Rightarrow$ $d-u \leq \operatorname{rk}(A)$ for $A \neq B \in \mathcal{C}$.

Assume there are two distinct matrices $M$ and $M^{\prime}$ in $\mathcal{C}$ with $\operatorname{rk}(M)<d / 2$ and $\operatorname{rk}\left(M^{\prime}\right)<d / 2$, then again by Inequality (5), we have $d \leq \operatorname{rk}\left(M-M^{\prime}\right) \leq \operatorname{rk}(M)+$ $\operatorname{rk}\left(-M^{\prime}\right)<d / 2+d / 2$, a contradiction.

Inequality (5) $\operatorname{shows~} \operatorname{rk}(X-Y) \leq \operatorname{rk}(X)+\operatorname{rk}(-Y) \leq 2 u$.

The next lemma is needed in Theorem 12 to complete the case of " $d=2 u$ ".

Lemma 11. Let $A_{1}, B_{1} \in \mathbb{F}_{q}^{a \times u}$ and $A_{2}, B_{2} \in \mathbb{F}_{q}^{u \times b}$. Then $\operatorname{rk}\left(A_{1} A_{2}-B_{1} B_{2}\right)=2 u$ iff $\operatorname{rk}\left(A_{1} B_{1}\right)=\operatorname{rk}\left(\begin{array}{l}A_{2} \\ B_{2}\end{array}\right)=2 u$.

Proof. We have $A_{1} A_{2}-B_{1} B_{2}=\left(\begin{array}{ll}A_{1} B_{1}\end{array}\right)\left(\begin{array}{c}A_{2} \\ -B_{2}\end{array}\right)=M$. Inequality (5) shows $\operatorname{rk}(M) \leq$ $\operatorname{rk}\left(A_{1} A_{2}\right)+\operatorname{rk}\left(B_{1} B_{2}\right) \leq u+u$ and Inequality (6) implies

$$
\begin{aligned}
& \operatorname{rk}\left(\begin{array}{ll}
A_{1} & B_{1}
\end{array}\right)+\operatorname{rk}\left(\begin{array}{c}
A_{2} \\
-B_{2}
\end{array}\right)-2 u \\
\leq & \operatorname{rk}(M) \leq \min \left\{\operatorname{rk}\left(\begin{array}{ll}
A_{1} B_{1}
\end{array}\right), \operatorname{rk}\left(\begin{array}{c}
A_{2} \\
-B_{2}
\end{array}\right)\right\} .
\end{aligned}
$$

If $\operatorname{rk}\left(\begin{array}{ll}A_{1} & B_{1}\end{array}\right)=\operatorname{rk}\left(\begin{array}{l}A_{2} \\ B_{2}\end{array}\right)=2 u$, then this shows $2 u \leq \operatorname{rk}(M)$. If $\operatorname{rk}\left(A_{1} B_{1}\right)<2 u$ or $\operatorname{rk}\left(\begin{array}{l}A_{2} \\ B_{2}\end{array}\right)<2 u$, then this shows $\operatorname{rk}(M)<2 u$.

We can settle the size of $\Lambda(q, a, b, d, u)$ for many parameters, in particular for all parameters with $u=1$ or all parameters with $d=2 u$.

Theorem 12. 1. If $2 u<d$ or if $\min \{a, b\}<d$, then $\Lambda(q, a, b, d, u)=1$.

2. If $\min \{a, b\} \leq u$, then $\Lambda(q, a, b, d, u)=M(q, a, b, d)$.

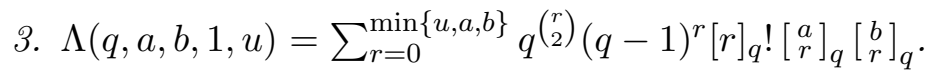

4. $\Lambda(q, a, b, 2 u, u)=A_{q}(\min \{a, b\}, 2 u ; u)$. 
Proof. 1. Lemma 10 implies the first case with the fact that $\mathcal{C}=\{0\}$ is an $(a \times$ $b, N, d ; u)_{q} \mathrm{RMC}$ of maximum cardinality. If $\min \{a, b\}<d$, then $d \leq \mathrm{d}_{\mathrm{r}}(A, B)=$ $\operatorname{rk}(A-B) \leq \min \{a, b\}$ implies also that $\mathcal{C}=\{0\}$ is of maximum cardinality.

2. If $\min \{a, b\} \leq u$, then $u$ imposes no restriction and any $(a \times b, N, d ; u)_{q} \mathrm{RMC}$, and the latter is an $(a \times b, N, d)_{q}$ RMC.

3. If $d=1$, then the unique maximum cardinality code consists of all matrices of rank at most $u$, so that Theorem 4 completes the proof.

4. The statement is true for $\min \{a, b\}<2 u$ by (1), so we assume $2 u \leq \min \{a, b\}$. Let $\mathcal{C}$ be an $(a \times b, N, 2 u ; u)_{q}$ RMC of size at least two. Lemma 10 implies that any matrix in $\mathcal{C}$ has rank $u$ and $\mathrm{d}_{\mathrm{r}}(A, B)=2 u$ for $A \neq B \in \mathcal{C}$.

Any matrix of rank $u$ in $\mathcal{C}$ is a product $A B$ for $A \in \mathbb{F}_{q}^{a \times u}$ and $B \in \mathbb{F}_{q}^{u \times b}$, both of full rank $u$. Hence, by Lemma 11, we have $\mathrm{d}_{\mathrm{r}}\left(A_{1} B_{1}, A_{2} B_{2}\right)=2 u$ iff $\mathrm{rk}\left(A_{1} B_{1}\right)=$ $\operatorname{rk}\left(\begin{array}{c}A_{2} \\ B_{2}\end{array}\right)=2 u$.

Let wlog. $b \leq a$, then choose $Y \subseteq \mathbb{F}_{q}^{u \times b}$ such that $Y$ consists of full rank matrices such that $\operatorname{rk}\left(\begin{array}{c}B_{1} \\ B_{2}\end{array}\right)=2 u$ for $B_{1} \neq B_{2} \in Y$, i.e., $\tau^{-1}(Y)$ is a $(b, \# Y, 2 u ; u)_{q} \mathrm{CDC}$ and the subspace distance is precisely $2 u \leq \mathrm{d}_{\mathrm{S}}\left(\tau^{-1}\left(B_{1}\right), \tau^{-1}\left(B_{2}\right)\right)=2\left(\operatorname{rk}\left(\begin{array}{c}B_{1} \\ B_{2}\end{array}\right)-u\right) \Leftrightarrow$ $2 u \leq \operatorname{rk}\left(\begin{array}{c}B_{1} \\ B_{2}\end{array}\right)$ by Equation (11). Hence, the maximum cardinality of $Y$ is $A_{q}(b, 2 u ; u)$. Choose $X \subseteq \mathbb{F}_{q}^{a \times u}$ such that $X$ consists of full rank matrices such that $\mathrm{rk}\left(A_{1} A_{2}\right)=$ $2 u$ for $A_{1} \neq A_{2} \in X$, as before $\tau^{-1}\left(X^{T}\right)$ is an $(a, \# X, 2 u ; u)_{q}$ CDC, where $X^{T}=$ $\left\{A^{T} \mid A \in X\right\}$. We choose $X$ of size $A_{q}(b, 2 u ; u) \leq A_{q}(a, 2 u ; u)$.

Each bijection $f: X \rightarrow Y$ gives then an $(a \times b, N, 2 u ; u)_{q}$ RMC of maximum cardinality $A_{q}(b, 2 u ; u)$, i.e., $\{x f(x) \mid x \in X\}$.

Note that the proof of (4) in Theorem 12 shows first that we have a constant-rank RMC in the case of $d=2 u$, so that e.g. [12, Theorem 2] could also complete the proof.

Theorem 12 shows that Inequality(77) and Theorem 9 can be arbitrarily bad, in fact, we have

$$
\begin{aligned}
\Delta(q, a, b, 2,1)= & 1 \in \Theta(1), \\
\Lambda(q, a, b, 2,1)= & {[\min \{a, b\}]_{q} \in \Theta\left(q^{\min \{a, b\}-1}\right), } \\
\Lambda(q, a, b, 2,1) \leq & 1+(q-1)[\min \{a, b\}-1]_{q}[\max \{a, b\}]_{q} \\
& \in \Theta\left(q^{a+b-2}\right), \text { and } \\
M(q, a, b, 2)= & q^{\max \{a, b\}(\min \{a, b\}-1)} \in \Theta\left(q^{a b-\max \{a, b\}}\right),
\end{aligned}
$$

for $2 \leq \min \{a, b\}$, using the Landau- $\Theta$ and Lemma 1 . 
Corollary 13. For $1 \leq d$ and $0 \leq i \leq 2 u-d$, we have

$$
\begin{aligned}
\Lambda(q, a, b, d, u) & \geq \Lambda(q, a+i, b+2 u-d-i, 2 u, u) \\
& =A_{q}(\min \{a+i, b+2 u-d-i\}, 2 u ; u)
\end{aligned}
$$

which yields the strongest bound for

$$
i=\max \{0, \min \{2 u-d,\lfloor(b-a-d) / 2\rfloor+u\}\} .
$$

Proof. Let $\mathcal{C}$ be an $((a+i) \times(b+2 u-d-i), N, 2 u ; u)_{q}$ RMC. We apply the puncturing argument of Theorem $9 i$ times to the rows and $2 u-d-i$ to the columns of any matrix in $\mathcal{C}$ to obtain an $(a \times b, N, d ; u)_{q}$ RMC $\mathcal{C}^{\prime}$. Choosing $\mathcal{C}$ of maximum size shows the first inequality. Next, (4) in Theorem 12 shows the equality. The optimal choice of $i$ follows by $A_{q}(v, d ; k) \leq A_{q}(v+1, d ; k)$ and $a+i=b+2 u-d-i \Leftrightarrow i=(b-a-d) / 2+u$.

Corollary 13 is sometimes stronger than Inequality (7), e.g. $\Lambda(q, 5,5,3,2) \geq 1$ by Inequality (7) but $\Lambda(q, 5,5,3,2) \geq A_{q}(5,4 ; 2)=q^{3}+1$ by Corollary 13, using $i=0$ and the equality follows from [1].

We present another lower bound of $\Lambda(q, a, b, d, u)$ involving CDCs and need therefore the following lemma.

Lemma 14. Let $A_{1}, B_{1} \in \mathbb{F}_{q}^{a \times u}$ and $A_{2}, B_{2} \in \mathbb{F}_{q}^{u \times b}$ with $\operatorname{rk}\left(A_{1}\right)=\operatorname{rk}\left(A_{2}\right)=\operatorname{rk}\left(B_{1}\right)=$ $\operatorname{rk}\left(B_{2}\right)=u$. Then

$$
\begin{aligned}
& \mathrm{d}_{\mathrm{s}}\left(\tau^{-1}\left(A_{1}^{T}\right), \tau^{-1}\left(B_{1}^{T}\right)\right)+\mathrm{d}_{\mathrm{s}}\left(\tau^{-1}\left(A_{2}\right), \tau^{-1}\left(B_{2}\right)\right) \\
\leq & 2 \mathrm{~d}_{\mathrm{r}}\left(A_{1} A_{2}, B_{1} B_{2}\right) .
\end{aligned}
$$

Proof. Using Equation (11), we have

$$
\begin{aligned}
& d_{1}=\mathrm{d}_{\mathrm{S}}\left(\tau^{-1}\left(A_{1}^{T}\right), \tau^{-1}\left(B_{1}^{T}\right)\right)=2\left(\operatorname{rk}\left(\begin{array}{c}
A_{1}^{T} \\
B_{1}^{T}
\end{array}\right)-u\right) \\
= & 2\left(\operatorname{rk}\left(A_{1} B_{1}\right)-u\right) \Leftrightarrow \operatorname{rk}\left(\begin{array}{ll}
A_{1} & B_{1}
\end{array}\right)=d_{1} / 2+u
\end{aligned}
$$

and

$$
\begin{aligned}
& d_{2}=\mathrm{d}_{\mathrm{s}}\left(\tau^{-1}\left(A_{2}\right), \tau^{-1}\left(B_{2}\right)\right)=2\left(\operatorname{rk}\left(\begin{array}{c}
A_{2} \\
B_{2}
\end{array}\right)-u\right) \\
= & 2\left(\operatorname{rk}\left(\begin{array}{c}
A_{2} \\
-B_{2}
\end{array}\right)-u\right) \Leftrightarrow \operatorname{rk}\left(\begin{array}{c}
A_{2} \\
-B_{2}
\end{array}\right)=d_{2} / 2+u .
\end{aligned}
$$

Then Inequality (6) and

$$
\begin{aligned}
& 2 \mathrm{~d}_{\mathrm{r}}\left(A_{1} A_{2}, B_{1} B_{2}\right)=2 \operatorname{rk}\left(\left(A_{1} B_{1}\right)\left(\begin{array}{c}
A_{2} \\
-B_{2}
\end{array}\right)\right) \\
\geq & 2\left(\operatorname{rk}\left(\left(A_{1} B_{1}\right)\right)+\operatorname{rk}\left(\left(\begin{array}{c}
A_{2} \\
-B_{2}
\end{array}\right)\right)-2 u\right) \\
= & 2\left(d_{1} / 2+u+d_{2} / 2+u-2 u\right)=d_{1}+d_{2}
\end{aligned}
$$

complete the proof. 
Theorem 15. Let $2 d \leq d_{1}+d_{2}$, then $\Lambda(q, a, b, d, u) \geq \min \left\{A_{q}\left(a, d_{1} ; u\right), A_{q}\left(b, d_{2} ; u\right)\right\}$.

Proof. Choose $\mathcal{Y}$ as a $\left(b, N, d_{1} ; u\right)_{q}$ CDC and $\mathcal{X}$ as an $\left(a, N, d_{2} ; u\right)_{q}$ CDC with $2 d \leq d_{1}+d_{2}$.

Let $Y \subseteq \mathbb{F}_{q}^{u \times b}$ such that $Y$ consists of full rank matrices and for each $U \in \mathcal{Y}$ there is exactly one $y \in Y$ with $\tau^{-1}(y)=U$.

Let $X \subseteq \mathbb{F}_{q}^{a \times u}$ such that $X$ consists of full rank matrices and for each $W \in \mathcal{X}$ there is exactly one $x \in X$ with $\tau^{-1}\left(x^{T}\right)=W$.

In particular, $\# Y=\# X=N$.

Then, each bijection $f: X \rightarrow Y$ gives then an $(a \times b, N, d ; u)_{q}$ RMC of cardinality $N$, i.e., $\mathcal{C}=\{x f(x) \mid x \in X\}$, because for $A_{1} A_{2} \neq B_{1} B_{2} \in \mathcal{C}$ such that $A_{1}, B_{1} \in \mathcal{X}$ and $A_{2}, B_{2} \in \mathcal{Y}$, Lemma 14 implies

$$
\begin{gathered}
2 d \leq d_{1}+d_{2} \leq \mathrm{d}_{\mathrm{s}}\left(\tau^{-1}\left(A_{1}^{T}\right), \tau^{-1}\left(B_{1}^{T}\right)\right) \\
+\mathrm{d}_{\mathrm{s}}\left(\tau^{-1}\left(A_{2}\right), \tau^{-1}\left(B_{2}\right)\right) \leq 2 \mathrm{~d}_{\mathrm{r}}\left(A_{1} A_{2}, B_{1} B_{2}\right)
\end{gathered}
$$

and each matrix $x f(x) \in \mathcal{C}$ has exactly the rank $u$.

Since we are actually constructing only a subset of an RRMC which is a constant-rank RMC in Theorem 15, [12, Proposition 3] could also be applied.

Note, that Theorem 15 implies the lower bound of the equality in (4) of Theorem 12, i.e., if $d=2 u=d_{1}=d_{2}$, then

$$
\begin{aligned}
& \Lambda(q, a, b, 2 u, u) \\
\geq & \min \left\{A_{q}(a, 2 u ; u), A_{q}(b, 2 u ; u)\right\}=A_{q}(\min \{a, b\}, 2 u ; u) .
\end{aligned}
$$

\section{Previous linkage constructions}

All constructions in this section but Theorem 18 can be proved by Lemma 23 which then implies supersets of parameters as the original proofs. Note, that we also allow $\{0\}$ as RMC.

The original linkage construction was independently discovered by Gluesing-Luerssen and Troha in [15] and by Silberstein and Horlemann-Trautmann in [25].

Special cases were already used by Gluesing-Luerssen, Morrison, and Troha in [14, Theorem 5.1] for cyclic orbit codes and by Etzion and Vardy in [9, Theorem 11] for spreads.

Theorem 16 ( [15, Theorem 2.3] and [25, Theorem 37 and Corollary 39]). Let $d / 2, k, r, s$ be integers with $2 \leq d / 2 \leq k, k \leq r$, and $k \leq s$. Let $\mathcal{A}$ be an $(r, \# \mathcal{A}, d ; k)_{q} C D C$ and $\mathcal{B}$ be an $(s, \# \mathcal{B}, d ; k)_{q} C D C$. Let $\mathcal{M}$ be $a(k \times s, \# \mathcal{M}, d / 2)_{q} R M C$. Then

$$
\begin{aligned}
& \left\{\tau^{-1}(\tau(A) \mid M): A \in \mathcal{A}, M \in \mathcal{M}\right\} \\
\cup & \left\{\tau^{-1}(0 \mid \tau(B)): B \in \mathcal{B}\right\}
\end{aligned}
$$

is an $(r+s, \# \mathcal{A} \cdot \# \mathcal{M}+\# \mathcal{B}, d ; k)_{q} C D C$.

In particular,

$$
A_{q}(r+s, d ; k) \geq A_{q}(r, d ; k) \cdot M(q, k, s, d / 2)+A_{q}(s, d ; k) .
$$


In [20], Heinlein and Kurz combined Theorem 16] with Lemma 2 to get the following so-called improved linkage construction.

Theorem 17 ( [20, Theorem 18], cf. [17, Theorem 136]). Let $d / 2, k, r, s, t$ be integers with $2 \leq d / 2 \leq k \leq(r+s) / 2, k \leq r, k \leq s+t$, and $0 \leq t \leq k-d / 2$. Let $\mathcal{A}$ be an $(r, \# \mathcal{A}, d ; k)_{q} C D C$ and $\mathcal{B}$ be an $(s+t, \# \mathcal{B}, d ; k)_{q} C D C$. Let $\mathcal{M}$ be $a(k \times s, \# \mathcal{M}, d / 2)_{q}$ RMC. Then

$$
\begin{aligned}
& \left\{\tau^{-1}(\tau(A) \mid M): A \in \mathcal{A}, M \in \mathcal{M}\right\} \\
\cup & \left\{\tau^{-1}(0 \mid \tau(B)): B \in \mathcal{B}\right\}
\end{aligned}
$$

is an $(r+s, \# \mathcal{A} \cdot \# \mathcal{M}+\# \mathcal{B}, d ; k)_{q} C D C$.

In particular,

$$
\begin{aligned}
& A_{q}(r+s, d ; k) \\
\geq & A_{q}(r, d ; k) \cdot M(q, k, s, d / 2)+A_{q}(s+k-d / 2, d ; k) .
\end{aligned}
$$

Theorem 17 was again improved by a generalized extension to any subcode of the form $\left\{\tau^{-1}(\tau(A) \mid M): A \in \mathcal{A}, M \in \mathcal{M}\right\}$ by Kurz in 22. Using the notation in [22, for $0 \leq w \leq v$ let $B_{q}(v, w, d ; k)$ be the maximum cardinality of a $(v, \# \mathcal{B}, d ; k)_{q} \operatorname{CDC~} \mathcal{B}$ such that there is a $w$-subspace $W$ with $\operatorname{dim}(W \cap B) \geq d / 2$ for each $B \in \mathcal{B}$.

Unfortunately, the quantity $B_{q}(v, w, d ; k)$ is not known in general as they generalize the numbers $A_{q}(v, d ; k)$ which are not well understood either, but [22] contains a lower bound:

Theorem 18 ( [22, Theorem 3.2, Proposition 4.1, and Theorem 4.2]). Let $d / 2, k, r, s$ be integers with $2 \leq d / 2 \leq k \leq(r+s) / 2, k \leq r$, and $d / 2 \leq s$.

1. Let $\mathcal{A}$ be an $(r, \# \mathcal{A}, d ; k)_{q} C D C$. Let $\mathcal{M}$ be a $(k \times s, \# \mathcal{M}, d / 2)_{q} R M C$. Then the $s$ subspace $W=\tau^{-1}(0 \mid I)$ intersects each codeword in $\left\{\tau^{-1}(\tau(A) \mid M): A \in \mathcal{A}, M \in\right.$ $\mathcal{M}\}$ trivial. Let $\mathcal{B}$ be an $(r+s, \# \mathcal{B}, d ; k)_{q} C D C$ such that $\operatorname{dim}(W \cap B) \geq d / 2$ for each $B \in \mathcal{B}$. Then

$$
\left\{\tau^{-1}(\tau(A) \mid M): A \in \mathcal{A}, M \in \mathcal{M}\right\} \cup \mathcal{B}
$$

is an $(r+s, \# \mathcal{A} \cdot \# \mathcal{M}+\# \mathcal{B}, d ; k)_{q} C D C$.

2.

$$
\begin{aligned}
& A_{q}(r+s, d ; k) \\
\geq & A_{q}(r, d ; k) \cdot M(q, k, s, d / 2)+B_{q}(r+s, s, d ; k)
\end{aligned}
$$

3. For $4 \leq k+1 \leq w+2 \leq v$ :

$$
B_{q}(v, w, 2 k-2 ; k) \geq A_{q}(w, 2 k-4 ; k-1)
$$


4. For $3 \leq k \leq s+1$ :

$$
\begin{aligned}
& A_{q}(r+s, 2 k-2 ; k) \\
\geq & A_{q}(r, 2 k-2 ; k) \cdot M(q, k, s, k-1)+A_{q}(s, 2 k-4 ; k-1)
\end{aligned}
$$

$\mathrm{Xu}$ and Chen developed in 27] a different direction as they incorporate matrices with lower and upper bounded ranks in a construction of CDCs.

Theorem 19 ( [27, Theorem 3]). Let $d / 2, k$ be integers with $2 \leq d / 2 \leq k$. Let $\mathcal{M}$ be a $(k \times k, \# \mathcal{M}, d / 2)_{q} R M C$ and let $\mathcal{R}$ be $a(k \times k, \# \mathcal{R}, d / 2 ; k-d / 2)_{q} R M C$. Then

$$
\left\{\tau^{-1}(I \mid M): M \in \mathcal{M}\right\} \cup\left\{\tau^{-1}(R \mid I): R \in \mathcal{R}\right\}
$$

is $a(2 k, \# \mathcal{M}+\# \mathcal{R}, d ; k)_{q} C D C$.

In particular,

$$
A_{q}(2 k, d ; k) \geq M(q, k, k, d / 2)+\Lambda(q, k, k, d / 2, k-d / 2)
$$

Finally, Theorem 19 was improved by Chen, He, Weng, and $\mathrm{Xu}$ in 2 ] by allowing the dimensions of the ambient spaces to vary. This is the so-called parallel linkage construction.

Theorem 20 ( [2, Theorem 3.1]). Let $d / 2, k, n$ be integers with $2 \leq d / 2 \leq k$ and $0 \leq n$. Let $\mathcal{A}$ be a $(k+n, \# \mathcal{A}, d ; k)_{q} C D C$ such that each $A \in \mathcal{A}$ is of the form $\tau(A)=\left(I \mid A^{\prime}\right)$, i.e., it is a lifted $R M C$, and $\mathcal{B}$ be an $(n+k, \# \mathcal{B}, d ; k)_{q} C D C$. Let $\mathcal{M}$ be $a(k \times k, \# \mathcal{M}, d / 2)_{q}$ $R M C$ and $\mathcal{R}$ be $a(k \times k, \# \mathcal{R}, d / 2 ; k-d / 2)_{q} R M C$. Then

$$
\begin{aligned}
& \left\{\tau^{-1}(\tau(A) \mid M): A \in \mathcal{A}, M \in \mathcal{M}\right\} \\
\cup & \left\{\tau^{-1}(R \mid \tau(B)): R \in \mathcal{R}, B \in \mathcal{B}\right\}
\end{aligned}
$$

is an $(n+2 k, \# \mathcal{A} \cdot \# \mathcal{M}+\# \mathcal{R} \cdot \# \mathcal{B}, d ; k)_{q} C D C$.

In particular,

$$
\begin{aligned}
& A_{q}(n+2 k, d ; k) \\
\geq & M(q, k, n, d / 2) \cdot M(q, k, k, d / 2) \\
+ & A_{q}(n+k, d ; k) \cdot \Lambda(q, k, k, d / 2, k-d / 2) .
\end{aligned}
$$

Of course, the concatenation of an RMC with an RMC is again an RMC. To be more precise, if $\mathcal{M}$ is an $(a \times b, \# \mathcal{M}, d)_{q} \mathrm{RMC}$ and $\mathcal{N}$ is an $(a \times c, \# \mathcal{N}, d)_{q} \mathrm{RMC}$, then $\{(M \mid N): M \in \mathcal{M}, N \in \mathcal{N}\}$ is an $(a \times(b+c), \# \mathcal{M} \cdot \# \mathcal{N}, d)_{q}$ RMC, since Inequality (4) implies

$$
\begin{aligned}
& \operatorname{rk}\left((M \mid N)-\left(M^{\prime} \mid N^{\prime}\right)\right)=\operatorname{rk}\left(M-M^{\prime} \mid N-N^{\prime}\right) \\
\geq & \max \left\{\operatorname{rk}\left(M-M^{\prime}\right), \operatorname{rk}\left(N-N^{\prime}\right)\right\} \geq d
\end{aligned}
$$

for $M, M^{\prime} \in \mathcal{M}$ and $N, N^{\prime} \in \mathcal{N}$ with $(M \mid N) \neq\left(M^{\prime} \mid N^{\prime}\right)$.

Hence, we can improve Theorem 20 to the following construction. 
Theorem 21. Let $d / 2, k, s$ be integers with $2 \leq d / 2 \leq k$ and $k \leq s$. Let $\mathcal{A}$ be a $(k+s, \# \mathcal{A}, d ; k)_{q} C D C$ such that each $A \in \mathcal{A}$ is of the form $\tau(A)=\left(I \mid A^{\prime}\right)$, i.e., it is a lifted $R M C$, and $\mathcal{B}$ be an $(s, \# \mathcal{B}, d ; k)_{q} C D C$. Let $\mathcal{R}$ be $a(k \times k, \# \mathcal{R}, d / 2 ; k-d / 2)_{q} R M C$. Then

$$
\mathcal{A} \cup\left\{\tau^{-1}(R \mid \tau(B)): R \in \mathcal{R}, B \in \mathcal{B}\right\}
$$

is $a(k+s, \# \mathcal{A}+\# \mathcal{R} \cdot \# \mathcal{B}, d ; k)_{q} C D C$.

In particular,

$$
\begin{aligned}
& A_{q}(k+s, d ; k) \\
\geq & M(q, k, s, d / 2)+A_{q}(s, d ; k) \cdot \Lambda(q, k, k, d / 2, k-d / 2) .
\end{aligned}
$$

Lemma 22. The bound in Theorem 21 is equivalent to the bound in Theorem 20 iff $k \leq n$ or $n=0$ and stronger iff $0<n<k$.

Proof. Note that $s=n+k$ and $0 \leq n \Leftrightarrow k \leq s$. We have

$$
\begin{aligned}
& \text { bound in Theorem } 21 \geq \text { bound in Theorem 20 } \\
\Leftrightarrow & M(q, k, s, d / 2) \geq M(q, k, n, d / 2) \cdot M(q, k, k, d / 2) \\
\Leftrightarrow & q^{s(k-d / 2+1)} \geq\left\lceil q^{\max \{n, k\}(\min \{n, k\}-d / 2+1)}\right\rceil \cdot q^{k(k-d / 2+1)} \\
\Leftrightarrow & q^{n(k-d / 2+1)} \geq\left\lceil q^{\max \{n, k\}(\min \{n, k\}-d / 2+1)}\right\rceil .
\end{aligned}
$$

If $k \leq n$, the exponent $\max \{n, k\}(\min \{n, k\}-d / 2+1)=n(k-d / 2+1)$ is at least one and both sides of the inequality coincide.

If $0 \leq n<\min \{d / 2, k\}$, the exponent $\max \{n, k\}(\min \{n, k\}-d / 2+1)=k(n-d / 2+1)$ is at most zero, so the right hand side of the inequality is one, while the left hand side is one iff $n=0$ and else greater than one.

If $d / 2 \leq n<k$, the exponent $\max \{n, k\}(\min \{n, k\}-d / 2+1)=k(n-d / 2+1)$ is at least $k$, so we continue:

$$
\begin{aligned}
& \Leftrightarrow q^{n(k-d / 2+1)} \geq q^{k(n-d / 2+1)} \\
& \Leftrightarrow n(k-d / 2+1) \geq k(n-d / 2+1) \\
& \Leftrightarrow n(-d / 2+1) \geq k(-d / 2+1) \\
& \Leftrightarrow(n-k)(-d / 2+1) \geq 0 .
\end{aligned}
$$

Due to $n<k$ and $2 \leq d / 2$, the left hand side is at least one, proving the statement.

If $\mathcal{A}$ in Theorem 21, $\mathcal{A}$ and $\mathcal{M}$ in Theorem 20 or $\mathcal{M}$ in Theorem 19 are chosen to be of maximum size, respectively, then they give rise to so-called lifted maximum rankdistance (LMRD) codes and any superset of this particular subcode is upper bounded by more elaborate bounds first proved by Etzion and Silberstein in [ 8 , Theorems 10 and 11] and improved by the author in [18, Theorem 1], cf. [17, Proposition 99]. To overcome this difficulty, we do not restrict this part of the construction to lifted maximum rankdistance codes in Theorem 25. 


\section{The generalized linkage construction}

Lemma 23. Let $k, r, s$ be positive integers and $A, C \in \mathbb{F}_{q}^{k \times r}$ and $B, D \in \mathbb{F}_{q}^{k \times s}$ be matrices such that $\operatorname{rk}(A \mid B)=\operatorname{rk}(C \mid D)=k$. If

1. $\operatorname{rk} A=\operatorname{rk} C=k$ and $d \leq \mathrm{d}_{\mathrm{s}}\left(\tau^{-1}(A), \tau^{-1}(C)\right)$,

2. $A=C$ and $\operatorname{rk} A=k$ and $d / 2 \leq \mathrm{d}_{\mathrm{r}}(B, D)$ or

3. $d / 2 \leq|\operatorname{rk} A-\operatorname{rk} C|$,

then

$$
\begin{aligned}
d & \leq \mathrm{d}_{\mathrm{s}}\left(\tau^{-1}(A \mid B), \tau^{-1}(C \mid D)\right) \\
& =\mathrm{d}_{\mathrm{s}}\left(\tau^{-1}(B \mid A), \tau^{-1}(D \mid C)\right) .
\end{aligned}
$$

Proof. For two subspaces $U$ and $W$ of dimension $k$ in a common vector space, we have with Equation (11)

$$
d \leq \mathrm{d}_{\mathrm{s}}(U, W) \Leftrightarrow \operatorname{rk}\left(\begin{array}{c}
\tau(U) \\
\tau(W)
\end{array}\right) \geq k+d / 2 .
$$

Since $\operatorname{rk}\left(\begin{array}{cc}M & N \\ O & P\end{array}\right)=\operatorname{rk}\left(\begin{array}{cc}N & M \\ P & O\end{array}\right)=\operatorname{rk}\left(\begin{array}{ll}\mathrm{R}\left(\begin{array}{ll}N & M\end{array}\right) \\ \mathrm{R}(P & O\end{array}\right)$ for any matrices $M, N, O, P$ with compatible sizes and ambient fields, we get

$$
\mathrm{d}_{\mathrm{s}}\left(\tau^{-1}(A \mid B), \tau^{-1}(C \mid D)\right)=\mathrm{d}_{\mathrm{s}}\left(\tau^{-1}(B \mid A), \tau^{-1}(D \mid C)\right) .
$$

The statement in question is $\operatorname{rk}\left(\begin{array}{cc}A & B \\ C & D\end{array}\right) \geq k+d / 2$.

1. Using Inequality (44), we obtain $\operatorname{rk}\left(\begin{array}{cc}A & B \\ C & D\end{array}\right) \geq \operatorname{rk}\left(\begin{array}{c}A \\ C\end{array}\right)$ and $\mathrm{d}_{\mathrm{s}}\left(\tau^{-1}(A), \tau^{-1}(C)\right) \geq d$ is equivalent to $\operatorname{rk}\left(\begin{array}{l}\mathrm{R}(A) \\ \mathrm{R}(C)\end{array}\right)=\operatorname{rk}\left(\begin{array}{c}A \\ C\end{array}\right) \geq k+d / 2$.

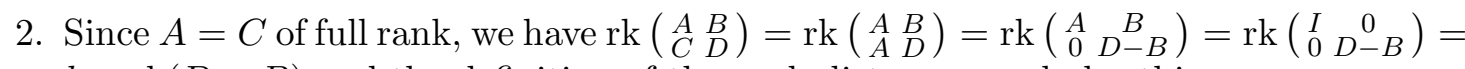
$k+\operatorname{rk}(D-B)$ and the definition of the rank-distance concludes this case.

3. Here, we use Lemma 2, Equality (2), Inequality (3), and let $[v](\{v\})$ denote the first $r$ (last $s$ ) entries in a vector $v$ so that $v=([v] \mid\{v\}), \mathrm{w}([\mathrm{p}(A \mid B)])=\operatorname{rk} A$, and $\mathrm{w}([\mathrm{p}(C \mid D)])=\operatorname{rk} C$. Hence,

$$
\begin{aligned}
& \mathrm{d}_{\mathrm{s}}\left(\tau^{-1}(A \mid B), \tau^{-1}(C \mid D)\right) \\
\geq & \mathrm{d}_{\mathrm{h}}\left(\mathrm{p}\left(\tau^{-1}(A \mid B)\right), \mathrm{p}\left(\tau^{-1}(C \mid D)\right)\right) \\
= & \mathrm{d}_{\mathrm{h}}(\mathrm{p}(A \mid B), \mathrm{p}(C \mid D)) \\
= & \mathrm{d}_{\mathrm{h}}([\mathrm{p}(A \mid B)],[\mathrm{p}(C \mid D)]) \\
+ & \mathrm{d}_{\mathrm{h}}(\{\mathrm{p}(A \mid B)\},\{\mathrm{p}(C \mid D)\}) \\
\geq & |\mathrm{w}([\mathrm{p}(A \mid B)])-\mathrm{w}([\mathrm{p}(C \mid D)])| \\
+ & |\mathrm{w}(\{\mathrm{p}(A \mid B)\})-\mathrm{w}(\{\mathrm{p}(C \mid D)\})| \\
= & |\operatorname{rk} A-\operatorname{rk} C|+|(k-\operatorname{rk} A)-(k-\operatorname{rk} C)| \\
= & 2|\operatorname{rk} A-\operatorname{rk} C|
\end{aligned}
$$

shows that $|\operatorname{rk} A-\operatorname{rk} C| \geq d / 2$ implies the minimum distance. 
Lemma 23 can of course be generalized to at least two blocks and this is used in Theorem 27.

Lemma 24. Let $k, m, n_{i}$ be positive integers and $A_{i}, B_{i} \in \mathbb{F}_{q}^{k \times n_{i}}$ be matrices such that $\operatorname{rk}\left(A_{1}|\ldots| A_{m}\right)=\operatorname{rk}\left(B_{1}|\ldots| B_{m}\right)=k(1 \leq i \leq m)$. If

1. $\operatorname{rk} A_{i}=\operatorname{rk} B_{i}=k$ and $d \leq \mathrm{d}_{\mathrm{s}}\left(\tau^{-1}\left(A_{i}\right), \tau^{-1}\left(B_{i}\right)\right)$,

2. $A_{i}=B_{i}$ and $\mathrm{rk} A_{i}=k$ and $d / 2 \leq \mathrm{d}_{\mathrm{r}}\left(\left(A_{1}|\ldots| A_{i-1}\left|A_{i+1}\right| \ldots \mid A_{m}\right),\left(B_{1}|\ldots|\right.\right.$ $\left.\left.B_{i-1}\left|B_{i+1}\right| \ldots \mid B_{m}\right)\right)$ or

3. $d / 2 \leq\left|\operatorname{rk} A_{i}-\operatorname{rk} B_{i}\right|$,

for some $i \in\{1, \ldots, m\}$, then

$$
d \leq \mathrm{d}_{\mathrm{s}}\left(\tau^{-1}\left(A_{1}|\ldots| A_{m}\right), \tau^{-1}\left(B_{1}|\ldots| B_{m}\right)\right) .
$$

Proof. Since

$$
\operatorname{rk}\left(\begin{array}{c}
A_{\pi(1)}|\ldots| A_{\pi(m)} \\
B_{\pi(1)}|\ldots| B_{\pi(m)}
\end{array}\right)=\operatorname{rk}\left(\begin{array}{c}
A_{1}|\ldots| A_{m} \\
B_{1}|\ldots| B_{m}
\end{array}\right)
$$

for any permutation $\pi$ on $\{1, \ldots, m\}$ we can by Equation (1) assume that $i=1$.

Then all three statements follow by the corresponding three statements in Lemma 23 using $r=n_{1}, s=n_{2}+\ldots+n_{m}, A=A_{1}, B=\left(A_{2}|\ldots| A_{m}\right), C=B_{1}$, and $D=\left(B_{2}|\ldots| B_{m}\right)$.

We use Lemma 23 to generalize Theorem 17 and Theorem 21 in a single construction.

Theorem 25. Let $d / 2, k, r, s, t$ be integers with $2 \leq d / 2 \leq k \leq(r+s) / 2, k \leq r, k \leq s+t$, and $0 \leq t \leq k-d / 2$. Let $\mathcal{A}$ be an $(r, \# \mathcal{A}, d ; k)_{q} C D C$ and $\mathcal{B}$ be an $(s+t, \# \mathcal{B}, d ; k)_{q} C D C$. Let $\mathcal{M}$ be a $(k \times s, \# \mathcal{M}, d / 2)_{q} R M C$ and $\mathcal{R}$ be $a(k \times(r-t), \# \mathcal{R}, d / 2 ; k-d / 2-t)_{q} R M C$. Then

$$
\begin{aligned}
&\left\{\tau^{-1}(\tau(A) \mid M): A \in \mathcal{A}, M \in \mathcal{M}\right\} \\
& \cup\left\{\tau^{-1}(R \mid \tau(B)): R \in \mathcal{R}, B \in \mathcal{B}\right\}
\end{aligned}
$$

is an $(r+s, \# \mathcal{A} \cdot \# \mathcal{M}+\# \mathcal{R} \cdot \# \mathcal{B}, d ; k)_{q} C D C$.

In particular,

$$
\begin{aligned}
& A_{q}(r+s, d ; k) \\
\geq & A_{q}(r, d ; k) \cdot M(q, k, s, d / 2) \\
+ & A_{q}(s+t, d ; k) \cdot \Lambda(q, k, r-t, d / 2, k-d / 2-t) .
\end{aligned}
$$


Proof. The distinctness of codewords follows from the minimum distance.

Let $A, A^{\prime} \in \mathcal{A}$ and $M, M^{\prime} \in \mathcal{M}$. If $A=A^{\prime}$, then (2) in Lemma 23 shows $d \leq$ $\mathrm{d}_{\mathrm{s}}\left(\tau^{-1}(\tau(A) \mid M), \tau^{-1}\left(\tau\left(A^{\prime}\right) \mid M^{\prime}\right)\right)$, else, i.e., $A \neq A^{\prime}$, then (1) in Lemma 23 shows the same statement.

Let $R, R^{\prime} \in \mathcal{R}$ and $B, B^{\prime} \in \mathcal{B}$. If $B=B^{\prime}$, then (2) in Lemma 23 shows $d \leq \mathrm{d}_{\mathrm{s}}\left(\tau^{-1}(R \mid\right.$ $\left.\tau(B)), \tau^{-1}\left(R^{\prime} \mid \tau\left(B^{\prime}\right)\right)\right)$, else, i.e., $B \neq B^{\prime}$, then (1) in Lemma 23 shows the same statement.

Let $A \in \mathcal{A}, M \in \mathcal{M}, R \in \mathcal{R}$, and $B \in \mathcal{B}$. By $[\tau(B)](\{\tau(B)\})$ we denote the first $t$ last s) columns of $\tau(B)$, in particular $\tau(B)=([\tau(B)] \mid\{\tau(B)\})$. Then, the condition in (3) in Lemma 23 is $d / 2 \leq|\operatorname{rk}(\tau(A))-\operatorname{rk}(R \mid[\tau(B)])|=|k-\operatorname{rk}(R \mid[\tau(B)])|=k-\operatorname{rk}(R \mid$ $[\tau(B)]) \Leftrightarrow \operatorname{rk}(R \mid[\tau(B)]) \leq k-d / 2$. By the choice of $\mathcal{R}$, we have $\operatorname{rk} R \leq k-d / 2-t$ and $\operatorname{rk}[\tau(B)] \leq t$, so that the statement follows with Inequality (4).

If $\mathcal{A}=\left\{\tau^{-1}(I)\right\}, r=k$, and $t=0$ is chosen in Theorem 25, we get Theorem 21 as special case and if $\mathcal{R}=\{0\}$ and $t=k-d / 2$, we get Theorem 17 as special case.

This fits also in the framework of Kurz [22], cf. Theorem 18, providing an alternative proof of Theorem 25.

Lemma 26. Let $d / 2, k, r, s, t$ be integers with $2 \leq d / 2 \leq k, 0 \leq r, k \leq s+t$, and $0 \leq$ $t \leq \min \{k-d / 2, r\}$. Let $\mathcal{B}$ be an $(s+t, \# \mathcal{B}, d ; k)_{q} C D C, \mathcal{R}$ be $a(k \times(r-t), \# \mathcal{R}, d / 2 ; k-$ $d / 2-t)_{q} R M C$, and $W=\tau(0 \mid I)$ of dimension $s$ in $\mathbb{F}_{q}^{r+s}$.

Then $\operatorname{dim}\left(W \cap \tau^{-1}(R \mid \tau(B))\right) \geq d / 2$ for all $R \in \mathcal{R}$ and $B \in \mathcal{B}$.

In particular,

$$
\begin{aligned}
& B_{q}(r+s, s, d ; k) \\
\geq & A_{q}(s+t, d ; k) \cdot \Lambda(q, k, r-t, d / 2, k-d / 2-t) .
\end{aligned}
$$

Proof. By $[\tau(B)](\{\tau(B)\})$ we denote the first $t$ (last $s$ ) columns of $\tau(B)$, in particular $\tau(B)=([\tau(B)] \mid\{\tau(B)\})$. Then we have with Inequality (4)

$$
\begin{aligned}
& \operatorname{dim}\left(W \cap \tau^{-1}(R \mid \tau(B))\right) \\
& =\operatorname{dim}(W)+\operatorname{dim}\left(\tau^{-1}(R \mid \tau(B))\right)-\operatorname{dim}\left(W+\tau^{-1}(R \mid \tau(B))\right) \\
& =s+k-\operatorname{dim}\left(\tau^{-1}(0|0| I)+\tau^{-1}(R|[\tau(B)]|\{\tau(B)\})\right) \\
& =s+k-\operatorname{rk}\left(\begin{array}{ccc}
0 & 0 & I \\
R & {[\tau(B)]} & I \\
\tau(B)
\end{array}\right) \\
& =s+k-\operatorname{rk}\left(\begin{array}{ccc}
0 & 0 & I \\
R & {[\tau(B)]} & 0
\end{array}\right)=k-\operatorname{rk}(R[\tau(B)]) \\
& \geq k-\operatorname{rk} R-\operatorname{rk}[\tau(B)] \geq k-(k-d / 2-t)-(t)=d / 2 \text {. }
\end{aligned}
$$

Then (1) and (2) in Theorem 18 together with Lemma 26 imply Theorem 25.

Independent to this paper, He developed in [16] a variation of the generalized linkage construction (Theorem 25). The construction [16, Theorem 2] arises as special case of Theorem 25] if $t=0$ and $\Lambda(q, k, r, d / 2, k-d / 2)$ is replaced by $\Delta(q, k, r, d / 2, k-d / 2)-1$. 
In particular, the lower bound provided by Theorem 25 is strictly better than the lower bound provided by [16, Theorem 2]. Furthermore, [16, Corollary 1] requires $k \geq d$, cf. [16, Section 4], in contrast to Theorem 25. In [16, Section 4], He asks for generalizations to $k \leq d$ and our generalized linkage construction (Theorem 25) provides an answer.

Note, that there are infinite families of parameters showing that the consideration of $t>0$ is justified. For example, consider $v=7=r+s, d=4$, and $k=3$. Then, the maximum cardinalities of the ingredients of the generalized linkage construction are well known and in the notation of Theorem 25:

\begin{tabular}{lll|llll|l}
$\mathrm{r}$ & $\mathrm{s}$ & $\mathrm{t}$ & $\# \mathcal{A}$ & $\# \mathcal{M}$ & $\# \mathcal{R}$ & $\# \mathcal{B}$ & $A_{q}(7,4 ; 3) \geq$ \\
\hline 3 & 4 & 0 & 1 & $q^{8}$ & {$[3]_{q}$} & 1 & $q^{8}+q^{2}+q+1$ \\
3 & 4 & 1 & 1 & $q^{8}$ & 1 & $q^{3}+1$ & $q^{8}+q^{3}+1$ \\
4 & 3 & 0 & 1 & $q^{6}$ & {$[3]_{q}$} & 1 & $q^{6}+q^{2}+q+1$ \\
4 & 3 & 1 & 1 & $q^{6}$ & 1 & 1 & $q^{6}+1$ \\
5 & 2 & 1 & $q^{3}+1$ & $q^{3}$ & 1 & 1 & $q^{6}+q^{3}+1$
\end{tabular}

Here, we use $A_{q}(5,4 ; 2)=q^{3}+1$ by [1], (1), and (4) of Theorem [12, In particular, the largest code constructed by the generalized linkage construction has cardinality $q^{8}+q^{3}+1$, uses $r=3, s=4$, and $t=1$, and its cardinality is strictly larger than the codes arising by choosing different parameters.

Independently to this paper, Cossidente, Kurz, Marino, and Pavese developed in [3, Lemma 4.1] a generalization of [2, Theorem 4.1] having the property that neither one of Theorem 25] and [3, Lemma 4.1] is a special case of the other. In the following theorem, we generalize both constructions in a single construction.

Theorem 27. Let $d / 2, k, m, n_{i}, t_{i}$ be integers with $2 \leq m, 2 \leq d / 2 \leq k \leq\left(\sum_{i=1}^{m} n_{i}\right) / 2$, $k \leq n_{i}+t_{i}$, and $0 \leq t_{i} \leq \min \left\{k-d / 2, n_{i-1}\right\} \quad(2 \leq i), t_{1}=0(1 \leq i \leq m)$. Let

- $\tau^{-1}\left(\mathcal{C}_{i}\right)$ be $\left(n_{i}+t_{i}, C_{i}, d ; k\right)_{q} C D C s$,

- $\mathcal{M}_{i}$ be $\left(k \times n_{i}, M_{i}, d / 2\right)_{q} R M C s$,

- $\mathcal{R}_{i}$ be $\left(k \times n_{i}, R_{i}, d / 2 ; k-d / 2\right)_{q}$ RRMCs,

- $\mathcal{S}_{i}$ be $\left(k \times\left(n_{i}-t_{i+1}\right), S_{i}, d / 2 ; k-d / 2-t_{i+1}\right)_{q} \operatorname{RRMCs}(i<m)$, and

- $\mathcal{S}_{m}$ be a set of size $S_{m}=1$ consisting of an empty matrix

for all $i \in\{1, \ldots, m\}$, then

$$
\begin{array}{r}
\bigcup_{i=1}^{m}\left\{\tau^{-1}\left(r_{1}|\ldots| r_{i-2}\left|s_{i-1}\right| c_{i}\left|m_{i+1}\right| \ldots \mid m_{m}\right)\right. \\
: r_{j} \in \mathcal{R}_{j} \quad(1 \leq j \leq i-2), s_{i-1} \in \mathcal{S}_{i-1}, c_{i} \in \mathcal{C}_{i}, \\
\left.m_{w} \in \mathcal{M}_{w} \quad(i+1 \leq w \leq m)\right\}
\end{array}
$$

is a $\left(\sum_{i=1}^{m} n_{i}, N, d ; k\right)_{q} C D C$ with

$$
N=\sum_{i=1}^{m} C_{i} \cdot S_{i} \cdot \prod_{j=1}^{i-2} R_{j} \cdot \prod_{w=i+1}^{m} M_{w} .
$$


In particular,

$$
\begin{aligned}
& A_{q}\left(\sum_{i=1}^{m} n_{i}, d ; k\right) \geq A_{q}\left(n_{1}, d ; k\right) \\
& \cdot \prod_{w=2}^{m} M\left(q, k, n_{w}, d / 2\right)+\sum_{i=2}^{m} A_{q}\left(n_{i}+t_{i}, d ; k\right) \\
& \cdot \Lambda\left(q, k, n_{i-1}-t_{i}, d / 2, k-d / 2-t_{i}\right) . \\
& \prod_{j=1}^{i-2} \Lambda\left(q, k, n_{j}, d / 2, k-d / 2\right) \cdot \prod_{w=i+1}^{m} M\left(q, k, n_{w}, d / 2\right) .
\end{aligned}
$$

Proof. The distinctness of codewords follows from the minimum distance.

Let $\tau^{-1}\left(r_{1}|\ldots| r_{i-2}\left|s_{i-1}\right| c_{i}\left|m_{i+1}\right| \ldots \mid m_{m}\right)$ and $\tau^{-1}\left(r_{1}^{\prime}|\ldots| r_{i-2}^{\prime}\left|s_{i-1}^{\prime}\right| c_{i}^{\prime} \mid\right.$ $\left.m_{i+1}^{\prime}|\ldots| m_{m}^{\prime}\right)$ be two distinct codewords in the same subcode.

If $c_{i}=c_{i}^{\prime}$, then (1) in Lemma 24 implies the minimum distance using the minimum subspace distance of $\mathcal{C}_{i}$. Else, by distinctness, there is a $1 \leq j \leq i-2$ with $r_{j} \neq r_{j}^{\prime}$ or $s_{i-1} \neq s_{i-1}^{\prime}$ or there is a $i+1 \leq w \leq m$ with $m_{w} \neq m_{w}^{\prime}$. We abbreviate all cases in $x \neq x^{\prime}$ for $x \in\left\{r_{j}, s_{i-1}, m_{w}\right\}$. Then, by the minimum rank distance and Inequality (4), we have $d / 2 \leq \mathrm{d}_{\mathrm{r}}\left(x, x^{\prime}\right) \leq \mathrm{d}_{\mathrm{r}}\left(\left(r_{1}|\ldots| r_{i-2}\left|s_{i-1}\right| m_{i+1}|\ldots| m_{m}\right),\left(r_{1}^{\prime}|\ldots| r_{i-2}^{\prime} \mid\right.\right.$ $\left.\left.s_{i-1}^{\prime}\left|m_{i+1}^{\prime}\right| \ldots \mid m_{m}^{\prime}\right)\right)$ so that (2) in Lemma 24 concludes this case.

Let $\tau^{-1}\left(r_{1}|\ldots| r_{i-2}\left|s_{i-1}\right| c_{i}\left|m_{i+1}\right| \ldots \mid m_{m}\right)$ and $\tau^{-1}\left(r_{1}^{\prime}|\ldots| r_{i^{\prime}-2}^{\prime}\left|s_{i^{\prime}-1}^{\prime}\right| c_{i^{\prime}}^{\prime} \mid\right.$ $\left.m_{i^{\prime}+1}^{\prime}|\ldots| m_{m}^{\prime}\right)$ be two distinct codewords in different subcodes corresponding to $i$ and $i^{\prime}$, we use without loss of generality $i<i^{\prime}$.

Define $x$ as $\left(s_{i^{\prime}-1}^{\prime} \mid\left[c_{i^{\prime}}^{\prime}\right]\right)$ if $i+1=i^{\prime}$, where $\left[c_{i^{\prime}}^{\prime}\right]$ is the matrix consisting of the leftmost $t_{i}$ columns of $c_{i^{\prime}}^{\prime}$, and as $r_{i}^{\prime}$ if $i+2 \leq i^{\prime}$. In the first case, we have $\operatorname{rk} x \leq$ $\operatorname{rk}\left(s_{i^{\prime}-1}^{\prime}\right)+\operatorname{rk}\left(\left[c_{i^{\prime}}^{\prime}\right]\right) \leq\left(k-d / 2-t_{i}\right)+\left(t_{i}\right)$ by Inequality (44), so that $\mathrm{rk} x \leq k-d / 2$ in both cases.

Then we have $\left|\operatorname{rk} c_{i}-\operatorname{rk} x\right|=\operatorname{rk} c_{i}-\operatorname{rk} x \geq k-(k-d / 2)=d / 2$ and (3) in Lemma 24 concludes this case.

This relates to other constructions as follows. If we set $m=2$, we obtain Theorem 25 , Using $t=0$, we get [3, Lemma 4.1]. If $m=s+1, n_{i}=n, t_{i}=0, d=2(n-t), k=n$, $\mathcal{C}_{i}=\left\{\tau^{-1}(I)\right\}$, we get [2, Theorem 4.1].

\section{New CDCs and better lower bounds}

According to the numerical evidence of http://subspacecodes.uni-bayreuth.de/, the generalized linkage construction (Theorem 25) increases all known lower bounds on $A_{q}(v, 4 ; 4)$ for all listed parameters $q$ and $12 \leq v$.

In the setting of $q=2$, the previously best known lower bound $A_{2}(12,4 ; 4) \geq 19664917$ is given by the improved linkage construction (Theorem [17), our new generalized linkage construction (Theorem 25) increases the bound to $A_{2}(12,4 ; 4) \geq 19673821$, while the parallel linkage construction (Theorem 20) only creates codes of size 19297741.

Hence, we compare the sizes of CDCs with $v=12$ and $d=k=4$ constructed by these three constructions for all $q$. 
The size of the code constructed in Theorem 25 using $r=8$ and $t=0$, so that $s=4$, is

$$
\begin{aligned}
& A_{q}(r, d ; k) \cdot M(q, k, s, d / 2) \\
+ & A_{q}(s+t, d ; k) \cdot \Lambda(q, k, r-t, d / 2, k-d / 2-t) \\
\geq & A_{q}(8,4 ; 4) \cdot M(q, 4,4,2)+A_{q}(4,4 ; 4) \cdot \Delta(q, 4,8,2,2) \\
= & A_{q}(8,4 ; 4) \cdot q^{4(4-2+1)}+1 \cdot\left(1+\left[{ }_{2}^{4}\right]_{q}\left(q^{8}-1\right)\right) \\
= & A_{q}(8,4 ; 4) \cdot q^{12}+1+\left[{ }_{2}^{4}\right]_{q}\left(q^{8}-1\right) \\
> & A_{q}(8,4 ; 4) \cdot q^{12}+\left[{ }_{2}^{4}\right]_{q}\left(q^{8}-1\right) \\
> & q^{12} \cdot q^{12}+q^{4}\left(q^{8}-1\right) \\
= & q^{24}+q^{12}-q^{4}
\end{aligned}
$$

For the last inequality, we use Theorem 6 and Lemma 1 .

We compare the generalized linkage construction to the parallel linkage construction. Unfortunately, the bound of Theorem 9, i.e., $\Lambda(q, 4,4,2,2) \leq q^{3}\left(q^{7}+q^{6}+q^{5}-q^{4}-q^{3}-\right.$ $\left.q^{2}+1\right)$, is too weak to show this result in general.

Lemma 28. If $2 \leq q$ is a prime power, $v=12$, and $d=k=4$, then Theorem 25 constructs a larger code than Theorem 21, utilizing $\Delta$ instead of $\Lambda$ in the latter.

Proof. The size of the code constructed in Theorem 21] uses $s=8$ and is, with $\Lambda$ replaced by $\Delta$,

$$
\begin{aligned}
& M(q, k, s, d / 2)+A_{q}(s, d ; k) \cdot \Delta(q, k, k, d / 2, k-d / 2) \\
= & M(q, 4,8,2)+A_{q}(8,4 ; 4) \cdot \Delta(q, 4,4,2,2) \\
= & q^{8(4-2+1)}+A_{q}(8,4 ; 4) \cdot\left(1+\left[\begin{array}{l}
4 \\
2
\end{array}\right]_{q}\left(q^{4}-1\right)\right)
\end{aligned}
$$

Due to Equation (8), we have

$$
\begin{aligned}
& q^{24}+A_{q}(8,4 ; 4)\left(1+\left[\begin{array}{c}
4 \\
2
\end{array}\right]_{q}\left(q^{4}-1\right)\right) \\
< & A_{q}(8,4 ; 4) q^{12}+1+\left[\begin{array}{c}
4 \\
2
\end{array}\right]_{q}\left(q^{8}-1\right) \\
\Leftrightarrow & q^{24}-1-\left[\begin{array}{l}
4 \\
2
\end{array}\right]_{q}\left(q^{8}-1\right)<A_{q}(8,4 ; 4)\left(q^{12}-1-\left[\begin{array}{c}
4 \\
2
\end{array}\right]_{q}\left(q^{4}-1\right)\right) .
\end{aligned}
$$

Using the lower bound of $A_{q}(8,4 ; 4)$ in Theorem 6 we will prove

$$
\begin{aligned}
& \frac{q^{24}-1-\left[\begin{array}{l}
4 \\
2
\end{array}\right]_{q}\left(q^{8}-1\right)}{q^{12}-1-\left[\begin{array}{l}
4 \\
2
\end{array}\right]_{q}\left(q^{4}-1\right)}<q^{12}+q^{2}\left(q^{2}+1\right)^{2}\left(q^{2}+q+1\right)+1 \\
\Leftrightarrow & \frac{q^{24}-1-\left(q^{2}+1\right)\left(q^{2}+q+1\right)\left(q^{8}-1\right)}{q^{12}-1-\left(q^{2}+1\right)\left(q^{2}+q+1\right)\left(q^{4}-1\right)} \\
< & q^{12}+q^{2}\left(q^{2}+1\right)^{2}\left(q^{2}+q+1\right)+1 \\
\Leftarrow & \frac{q^{24}-q^{2} q^{2}\left(q^{8}-1\right)}{q^{12}-1-\left(q^{2}+1\right)\left(q^{2}+q+1\right) q^{4}} \\
\leq & q^{12}+q^{2}\left(q^{2}+1\right)^{2}\left(q^{2}+q+1\right)
\end{aligned}
$$


which is equivalent to the nonnegativity of

$$
\begin{aligned}
& \left(q^{12}+q^{2}\left(q^{2}+1\right)^{2}\left(q^{2}+q+1\right)\right) \\
& \cdot\left(q^{12}-1-\left(q^{2}+1\right)\left(q^{2}+q+1\right) q^{4}\right)-\left(q^{24}-q^{2} q^{2}\left(q^{8}-1\right)\right) \\
= & q^{2}\left(q^{16}+q^{15}+q^{14}-q^{13}-5 q^{12}-8 q^{11}-13 q^{10}-12 q^{9}\right. \\
- & \left.13 q^{8}-8 q^{7}-7 q^{6}-3 q^{5}-4 q^{4}-2 q^{3}-4 q^{2}-q-1\right) \\
\geq & q^{2}\left(q^{16}+q^{15}+q^{14}-q^{13}-13 \sum_{i=0}^{12} q^{i}\right) \\
\geq & q^{2}\left(q^{16}+q^{15}+q^{14}-q^{13}-13 q^{13}\right) \\
= & q^{15}(q-2)\left(q^{2}+3 q+7\right) .
\end{aligned}
$$

Since the last term is nonnegative for $2 \leq q$, the statement follows.

We compare the generalized linkage construction to the improved linkage construction.

Lemma 29. If $2 \leq q$ is a prime power, $v=12$, and $d=k=4$, then Theorem 25 constructs a larger code than Theorem 17 .

Proof. The size of the code constructed in Theorem 17 using $t=2, s=12-r$, and $4 \leq r \leq 10$ is

$$
\begin{aligned}
& A_{q}(r, d ; k) \cdot M(q, k, s, d / 2)+A_{q}(s+k-d / 2, d ; k) \\
= & A_{q}(r, 4 ; 4) \cdot M(q, 4,12-r, 2)+A_{q}(14-r, d ; 4) \\
= & A_{q}(r, 4 ; 4) \cdot q^{\max \{12-r, 4\}(\min \{12-r, 4\}-1)}+A_{q}(14-r, 4 ; 4) \\
= & \begin{cases}A_{q}(r, 4 ; 4) \cdot q^{3(12-r)}+A_{q}(14-r, 4 ; 4) \quad \text { if } 4 \leq r \leq 8 \\
A_{q}(r, 4 ; 4) \cdot q^{4(11-r)}+A_{q}(14-r, 4 ; 4) & \text { if } 9 \leq r \leq 10\end{cases}
\end{aligned}
$$

Using Lemma 5 and Theorem 7 we have $A_{q}(4,4 ; 4)=A_{q}(4,4 ; 0)=1, A_{q}(5,4 ; 4)=$ $A_{q}(5,4 ; 1)=1, A_{q}(6,4 ; 4)=A_{q}(6,4 ; 2)=[6]_{q} /[2]_{q}=q^{4}+q^{2}+1$, and $A_{q}(7,4 ; 4)=$ $A_{q}(7,4 ; 3)$.

For $4 \leq x$, the Anticode bound in Theorem 8 and Lemma 1 imply $A_{q}(x, 4 ; 4) \leq$ $\left[\begin{array}{c}x \\ 4-2+1\end{array}\right]_{q} /\left[\begin{array}{c}4 \\ 4-2+1\end{array}\right]_{q}<4 q^{3(x-3)} / q^{3}=4 q^{3 x-12}$.

For $r=4$, Theorem 17 yields a CDC of size $q^{24}+A_{q}(10,4 ; 4)$. Then, comparing to Inequality (9), i.e.,

$$
\begin{aligned}
& A_{q}(8,4 ; 4) \cdot q^{12}+\left[{ }_{2}^{4}\right]_{q}\left(q^{8}-1\right)-q^{24}-A_{q}(10,4 ; 4) \\
\geq & \left(q^{12}+q^{2}\left(q^{2}+1\right)^{2}\left(q^{2}+q+1\right)+1\right) \cdot q^{12} \\
+ & \left(q^{8}-1\right)[4]_{q}[3]_{q} /[2]_{q}-q^{24}-[10]_{q}[9]_{q}[8]_{q} /\left([4]_{q}[3]_{q}[2]_{q}\right) \\
= & q^{20}+q^{19}+2 q^{18}+2 q^{17}+2 q^{16}-q^{14}-q^{13}-q^{12}-q^{11} \\
- & q^{10}-q^{9}-2 q^{8}-2 q^{7}-3 q^{6}-q^{5}-3 q^{4}-2 q^{3}-3 q^{2}-q-2 \\
> & q^{20}-3 \sum_{i=0}^{14} q^{i}=q^{20}-3[15]_{q}>q^{20}-3 q^{15}>0
\end{aligned}
$$


concludes this case.

For $r=5$, Theorem 17y yields a CDC of size $q^{21}+A_{q}(9,4 ; 4) \leq q^{21}+4 q^{15} \leq q^{21}+q^{17} \leq$ $q^{22}$, so that Inequality (10) concludes this case.

For $r=6$, Theorem 17 yields a CDC of size $\left([6]_{q} /[2]_{q}\right) q^{18}+A_{q}(8,4 ; 4) \leq 2 q^{22}+4 q^{12} \leq$ $q^{23}+q^{14} \leq q^{24}$, so that Inequality (10) concludes this case.

For $r=7$, Theorem 17 yields a CDC of size $A_{q}(7,4 ; 3)\left(q^{15}+1\right)$. Then, comparing to Inequality (9), i.e.,

$$
\begin{aligned}
& A_{q}(8,4 ; 4) q^{12}+\left[\begin{array}{l}
4 \\
2
\end{array}\right]_{q}\left(q^{8}-1\right)-A_{q}(7,4 ; 3)\left(q^{15}+1\right) \\
\geq & q^{24}+\left(q^{8}-1\right)[4]_{q}[3]_{q} /[2]_{q}-\left(q^{15}+1\right)[7]_{q}[6]_{q} /\left([3]_{q}[2]_{q}\right) \\
= & q^{24}-q^{23}-q^{21}-q^{20}-q^{19}-q^{18}-q^{17}-q^{15}+q^{12}+q^{11} \\
+ & 2 q^{10}+q^{9}-q^{6}-q^{5}-2 q^{4}-2 q^{3}-3 q^{2}-q-2 \\
> & q^{24}-q^{23}-q^{21}-3 \sum_{i=0}^{20} q^{i}=q^{24}-q^{23}-q^{21}-3[21]_{q} \\
> & q^{24}-q^{23}-4 q^{21}=(q-2)\left(q^{2}+q+2\right) q^{21} \geq 0,
\end{aligned}
$$

concludes this case.

For $r=8$, Theorem 17 yields a CDC of size $A_{q}(8,4 ; 4) q^{12}+[6]_{q} /[2]_{q}$. Then, comparing to Inequality (9), i.e.,

$$
\begin{aligned}
& A_{q}(8,4 ; 4) q^{12}+[6]_{q} /[2]_{q} \leq A_{q}(8,4 ; 4) \cdot q^{12}+\left[\begin{array}{l}
4 \\
2
\end{array}\right]_{q}\left(q^{8}-1\right) \\
\Leftrightarrow & {[6]_{q} /[2]_{q} \leq\left(q^{8}-1\right)[4]_{q}[3]_{q} /[2]_{q} } \\
\Leftrightarrow & {[6]_{q} \leq\left(q^{8}-1\right)[4]_{q}[3]_{q} } \\
\Leftarrow & q^{6}-1<q^{8}-1,
\end{aligned}
$$

concludes this case.

For $r=9$, Theorem 17 yields a CDC of size $A_{q}(9,4 ; 4) q^{8}+1 \leq 4 q^{23}+1$, so that Inequality (10) concludes this case for all $4 \leq q$. Next, we use $\left(\prod_{i=1}^{\infty}\left(1-3^{-i}\right)\right)^{-1}<1.8$ in Lemma 1, so that $A_{q}(9,4 ; 4) q^{8}+1 \leq 1.8 q^{23}+1$ and Inequality (10) concludes this case for $q=3$, too. Last, the Anticode bound is precisely $A_{2}(9,4 ; 4) \leq 52535$, so that $A_{2}(9,4 ; 4) 2^{8}+1 \leq 13448961<16781296=2^{24}+2^{12}-2^{4}$, concluding the case $r=9$ for all $q$.

For $r=10$, Theorem 17 yields a CDC of size $A_{q}(10,4 ; 4) q^{4}+1 \leq 4 q^{22}+1 \leq q^{24}+1$, so that Inequality (10) concludes this case.

\section{References}

[1] A. Beutelspacher. Partial spreads in finite projective spaces and partial designs. Math. Z., 145(3):211-229, 1975. 
[2] H. Chen, X. He, J. Weng, and L. Xu. New constructions of subspace codes using subsets of MRD codes in several blocks. arXiv:1908.03804, 2019.

[3] A. Cossidente, S. Kurz, G. Marino, and F. Pavese. Combining subspace codes. arXiv:1911.03387, 2019.

[4] A. Cossidente and F. Pavese. Subspace codes in $\mathrm{PG}(2 N-1, Q)$. Combinatorica, 37(6):1073-1095, 2017.

[5] J. de la Cruz, E. Gorla, H. H. López, and A. Ravagnani. Rank distribution of Delsarte codes. arXiv:1510.01008, 2015.

[6] P. Delsarte. Bilinear forms over a finite field, with applications to coding theory. $J$. Combin. Theory Ser. A, 25(3):226-241, 1978.

[7] T. Etzion and N. Silberstein. Error-correcting codes in projective spaces via rankmetric codes and Ferrers diagrams. IEEE Trans. Inform. Theory, 55(7):2909-2919, 2009.

[8] T. Etzion and N. Silberstein. Codes and designs related to lifted MRD codes. IEEE Trans. Inform. Theory, 59(2):1004-1017, 2013.

[9] T. Etzion and A. Vardy. Error-correcting codes in projective space. IEEE Trans. Inform. Theory, 57(2):1165-1173, 2011.

[10] S. D. Fisher and M. N. Alexander. Classroom Notes: Matrices over a Finite Field. Amer. Math. Monthly, 73(6):639-641, 1966.

[11] E. M. Gabidulin. Theory of codes with maximum rank distance. Problemy Peredachi Informatsii, 21(1):3-16, 1985.

[12] M. Gadouleau and Z. Yan. Constant-rank codes and their connection to constantdimension codes. IEEE Trans. Inform. Theory, 56(7):3207-3216, 2010.

[13] The GAP Group. GAP - Groups, Algorithms, and Programming, Version 4.10.0, 2019.

[14] H. Gluesing-Luerssen, K. Morrison, and C. Troha. Cyclic orbit codes and stabilizer subfields. Adv. Math. Commun., 9(2):177-197, 2015.

[15] H. Gluesing-Luerssen and C. Troha. Construction of subspace codes through linkage. Adv. Math. Commun., 10(3):525-540, 2016.

[16] X. He. Construction of Const Dimension Code from Two Parallel Versions of Linkage Construction. arXiv:1910.04472, 2019.

[17] D. Heinlein. Integer linear programming techniques for constant dimension codes and related structures. PhD thesis, Bayreuth, November 2018. 
[18] D. Heinlein. New LMRD code bounds for constant dimension codes and improved constructions. IEEE Trans. Inform. Theory, 65(8):4822-4830, 2019.

[19] D. Heinlein, M. Kiermaier, S. Kurz, and A. Wassermann. Tables of subspace codes. arXiv:1601.02864, 2016.

[20] D. Heinlein and S. Kurz. Asymptotic bounds for the sizes of constant dimension codes and an improved lower bound. In Coding theory and applications, volume 10495 of Lecture Notes in Comput. Sci., pages 163-191. Springer, Cham, 2017.

[21] R. Kötter and F. R. Kschischang. Coding for errors and erasures in random network coding. IEEE Trans. Inform. Theory, 54(8):3579-3591, 2008.

[22] S. Kurz. A note on the linkage construction for constant dimension codes. arXiv:1906.09780, 2019.

[23] G. Landsberg. Ueber eine Anzahlbestimmung und eine damit zusammenhängende Reihe. J. Reine Angew. Math., 111:87-88, 1893.

[24] S. Niskanen and P. R. J. Östergård. Cliquer User's Guide: Version 1.0. Helsinki University of Technology Helsinki, Finland, 2003.

[25] N. Silberstein and A.-L. Trautmann. Subspace codes based on graph matchings, Ferrers diagrams, and pending blocks. IEEE Trans. Inform. Theory, 61(7):39373953, 2015.

[26] H. Wang, C. Xing, and R. Safavi-Naini. Linear authentication codes: bounds and constructions. IEEE Trans. Inform. Theory, 49(4):866-872, 2003.

[27] L. Xu and H. Chen. New constant-dimension subspace codes from maximum rank distance codes. IEEE Trans. Inform. Theory, 64(9):6315-6319, 2018. 